The Free Internet Journal for Organic Chemistry
Paper

Arkivoc 2018, part ii, 205-214

\title{
Reaction of trihaloisocyanuric acids with alkynes: an efficient methodology for the preparation of $\beta$-haloenol acetates
}

\author{
Lívia T. C. Crespo, Geisa P. Nogueira, Marcio C. S. de Mattos,* and Pierre M. Esteves* \\ Instituto de Química, Departamento de Química Orgânica, Universidade Federal do Rio de Janeiro, \\ Cx Postal 68545, 21945-970, Rio de Janeiro, Brazil \\ Email: pesteves@iq.ufri.br, mmattos@iq.ufri.br
}

This paper is submitted in honor of Kenneth K. Laali

Received 06-07-2017

Accepted 10-31-2017

Published on line 12-03-2017

\section{Abstract}

The reaction between trihaloisocyanuric acids and alkynes in the presence of acetic acid provides an efficient methodology for preparation of $\beta$-haloenol acetates in yields ranging from 34 to $94 \%$, depending on the halogen and alkynes used. This methodology provides an alternative to typical procedures, which usually employ metal catalysis and are limited to terminal alkynes.

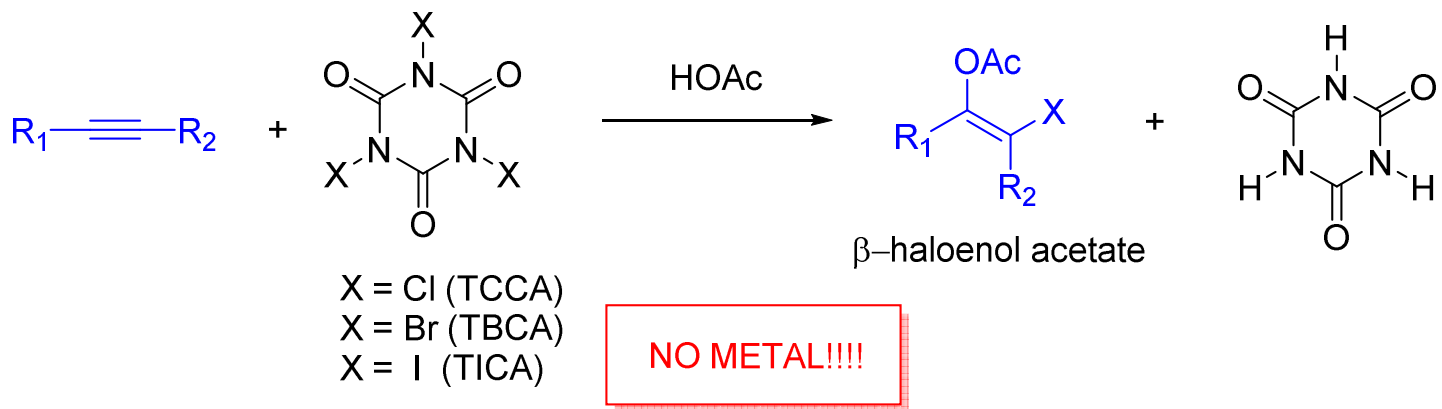

Keywords: Trihaloisocyanuric acids, $\beta$-haloenol acetates, electrophilic halogenation, alkynes 


\section{Introduction}

Trihaloisocyanuric acids (TXCA, Fig. 1) are inexpensive cyclic ureas used as versatile and green electrophilic halogenating agents. ${ }^{1-5}$ Among the haloisocyanuric acids, the most employed is the trichloroisocyanuric, or TCCA $(X=\mathrm{Cl})$, a stable solid used for swimming pool disinfection, sold in supermarkets or specialized stores on multigram/kilogram scale. ${ }^{6}$ Tribromoisocyanuric acid (TBCA, $X=B r$ ), although not yet widely commercially available, can be easily obtained from cyanuric acid, $\mathrm{KBr}$ and oxone, in a safe procedure. ${ }^{7}$ Triiodoisocyanuric acid (TICA, $X=I$ ) can be obtained from TCCA and $I_{2}$, heated in a sealed tube at high temperatures. ${ }^{8}$ Mixed trihaloisocyanuric acids are also known. ${ }^{9,10}$ All these reagents have been shown to be very efficient halenium $\left(\mathrm{X}^{+}\right)$releasing agents in diverse reactions, affording good to excellent yields of the desired halogenated products. Therefore, depending on the nature of the nucleophile reacting with these electrophilic agents, different products can be obtained, such as haloarenes, halohydrins, halocarbonyls, etc. ${ }^{1,5,6}$ The trihaloisocyanuric acids also are very interesting reagents from the Green Chemistry point of view, since they present good atom economy and are safe to be handled. After the reaction is completed, its by-product, the isocyanuric acid can be easily separated from the reaction media by simple filtration and can be reused for the synthesis of new TXCA batches. ${ }^{11}$
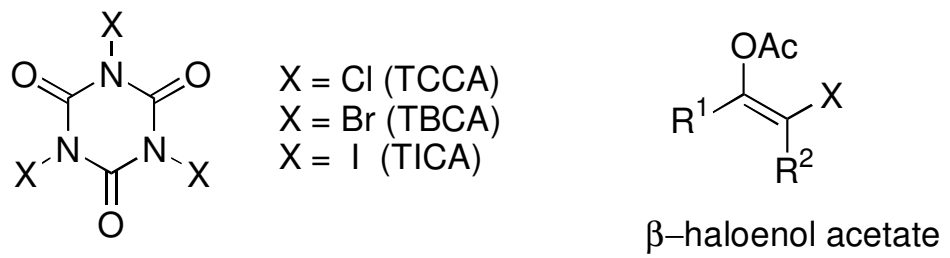

Figure 1. Structures of the trihaloisocyanuric acids and $\beta$-haloenol acetates.

$\beta$-Haloenol acetates (Figure 1) are key intermediates for diverse chemical transformations (Scheme 1), since they hold both enol acetate moiety as well as carbon-halogen reactivities, which can be explored in numerous synthetic ways. ${ }^{12}$ They can be prepared from an electrophilic halogen attack to alkynes, as demonstrated for instance by Barluenga et al., ${ }^{13}$ or by metallic catalysis using haloalkynes. ${ }^{12,14,15}$ Nevertheless, those methodologies usually need terminal haloalkynes and fancy metal catalysis, or are generally limited to iodo derivatives which shrink their synthetic scope. 


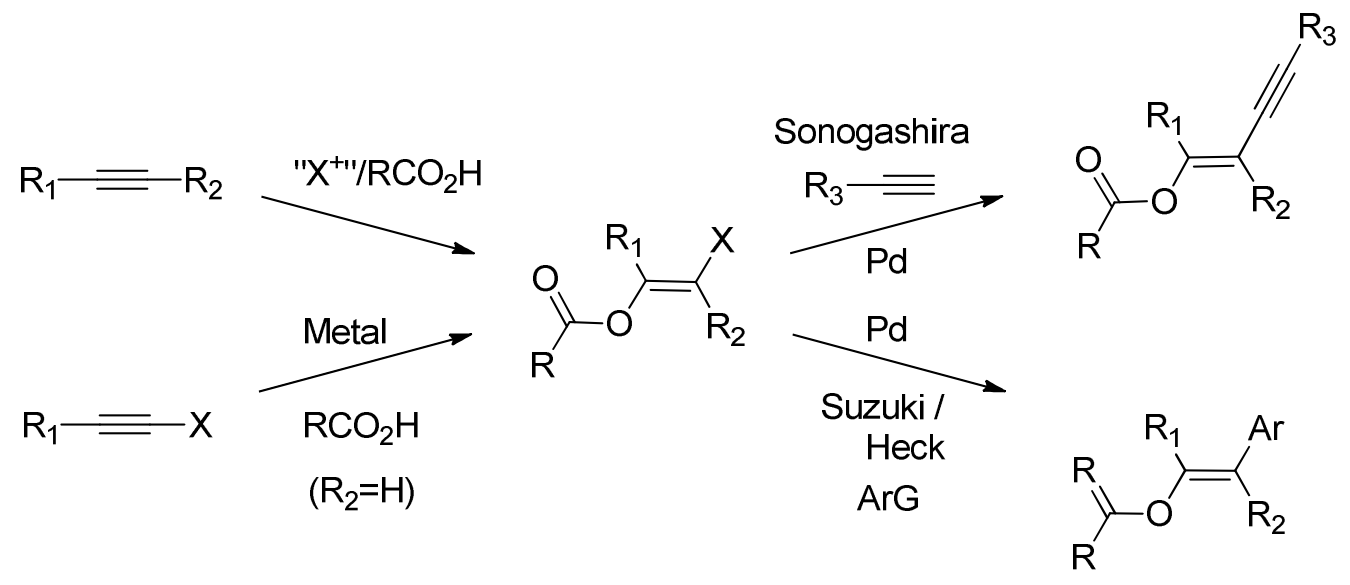

Scheme 1

Continuing our interest on the chemistry of trihaloisocyanuric acids, we have considered the use of TXCA as electrophilic reagent in the reaction with alkynes in order to prepare the interesting $\beta$-haloenol acetates, which are synthetically appealing building blocks. Herein we report the results of our researches.

\section{Results and Discussion}

In order to obtain the desired $\beta$-haloenol acetates, we have reacted selected alkynes with TXCA in the presence of acetic acid, according to the strategy shown in Scheme 2.

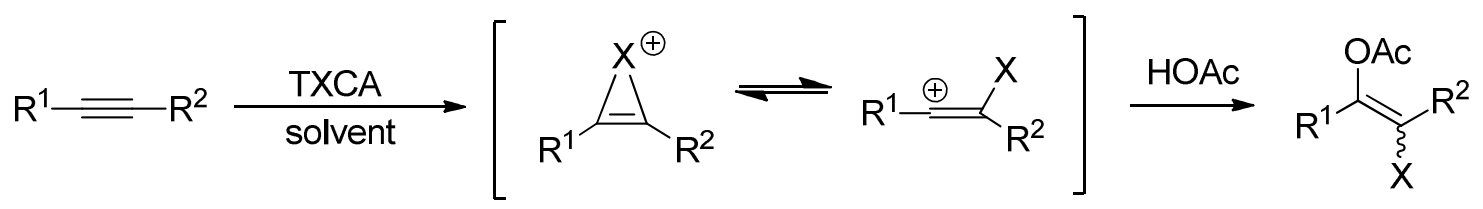

\section{Scheme 2}

Initially the reaction was carried out with TBCA and 1-phenyl-1-butyne (1 mol equiv.), in the presence of HOAc at room temperature, in order to optimize the reaction conditions, being the results shown in Table 1 . The use of HOAc as solvent (Table 1, entry 1 ) afforded the expected $\beta$-bromoenol acetate along with minor amount $(3 \%)$ of the corresponding $\beta$-dibromoketone, due to the hydrolysis of the products. In order to avoid this by-product we replaced $\mathrm{HOAc}$ by acetic anhydride $\left(\mathrm{Ac}_{2} \mathrm{O}\right)$, which afforded the desired product although in low conversion (Table 1, entry 2). Addition of 1 eq. of HOAc improved the yields, but still conversion was low (Table 1, entry 3). The use of $1: 1(\mathrm{v} / \mathrm{v})$ mixture of $\mathrm{Ac}_{2} \mathrm{O}$ and $\mathrm{HOAc}$ finally afforded only the $\beta$-bromoenol acetate in quantitative conversion after $1 \mathrm{~h}$ (Table 1 , entry 4 ) and $87 \%$ isolated yield. According to GC-MS and NMR analysis a mixture of $Z$ and $E$ diastereoisomers is formed under these conditions. Solvents with different polarities were used in order to evaluate the change in the ratio of these stereoisomers. The results show that in all cases approximately the same proportion of the $E$ and $Z$ stereoisomers is obtained regardless the solvent employed, only the conversion being affected by the solvent change. This suggests that the conjugated vinyl cation is being formed as intermediate instead of the corresponding bromonium ion (Scheme 3).

Table 1. Solvent influence on the reaction between TBCA and 1-phenyl-1-butyne 

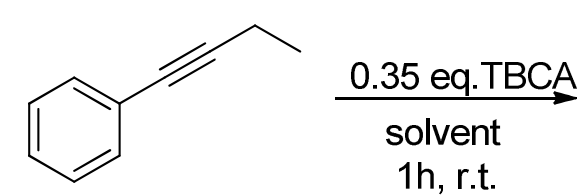<smiles>CCC(Br)=C(OC(C)=O)c1ccccc1</smiles>

\begin{tabular}{cccc}
\hline Entry & Solvent & Conversion (\%) $^{\mathrm{a}}$ & $\boldsymbol{E}: Z$ ratio \\
\hline 1 & $\mathrm{AcOH}$ & $97^{\mathrm{b}}$ & $88: 12$ \\
2 & $\mathrm{Ac}_{2} \mathrm{O}$ & $24^{\mathrm{c}}$ & $88: 12$ \\
3 & $\mathrm{Ac}_{2} \mathrm{O} / 1 \mathrm{eq} . \mathrm{AcOH}$ & 76 & $88: 12$ \\
4 & $\mathrm{Ac}_{2} \mathrm{O}: \mathrm{AcOH}(1: 1)$ & $100^{\mathrm{d}}$ & $85: 15$ \\
5 & $\mathrm{CH}_{2} \mathrm{Cl}_{2}$ & 34 & $87: 13$ \\
6 & $\mathrm{Hexane}^{\mathrm{n}}$ & $22^{\mathrm{e}}$ & $86: 14$ \\
\hline
\end{tabular}

${ }^{a}$ Determined by GC-MS; ${ }^{b} 3 \%$ of the corresponding dibromoketone formed; ${ }^{c}$ incomplete reaction; ${ }^{d} 87 \%$ isolated yield; ${ }^{\mathrm{e}} 65 \%$ after 4 days.

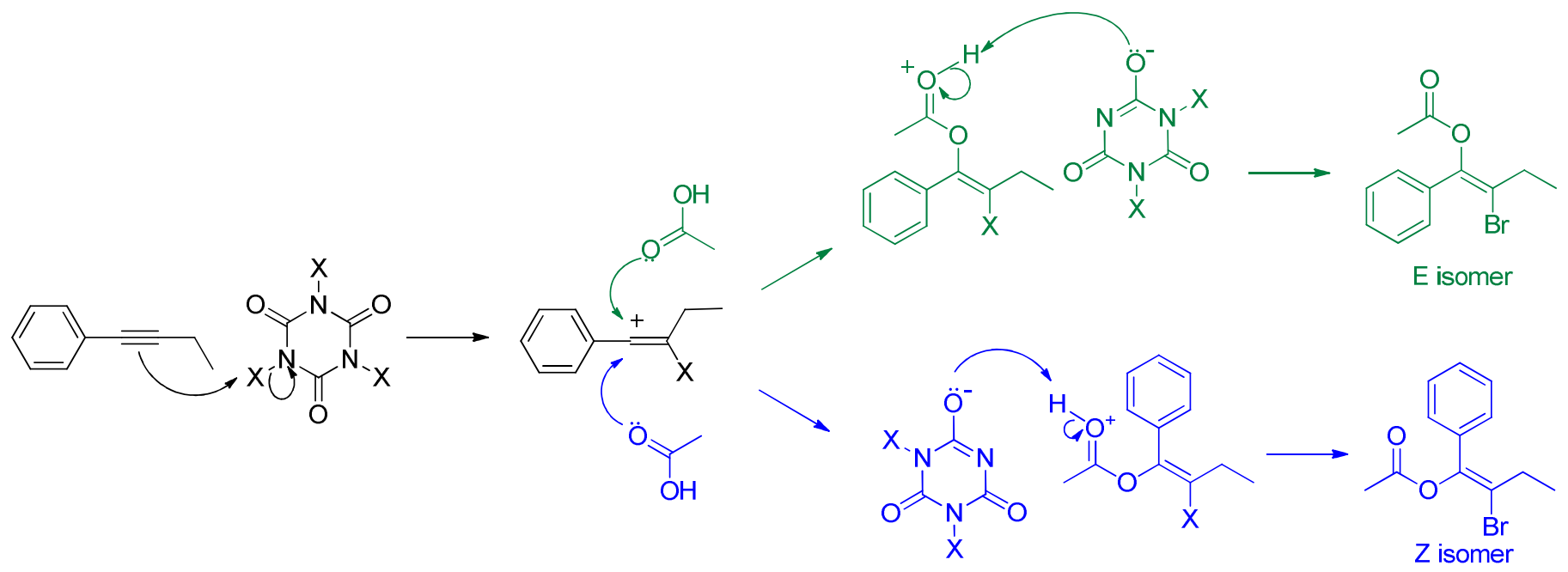

\section{Scheme 3}

Based upon this initial study we chose the 1:1 mixture of $\mathrm{HOAc}: \mathrm{Ac}_{2} \mathrm{O}$ as standard conditions for the reaction of TXCA with other alkynes $(2 \mathrm{mmol}$ of substrate, and $0.68 \mathrm{mmol}$ of TBCA in $6 \mathrm{~mL}$ of solvent at room temperature). The reaction was monitored by GC-MS until complete conversion of the substrate and in all cases the expected $\beta$-haloenol acetates were obtained, as shown in Table 2.

The reactions of alkynes with TBCA in $\mathrm{HOAc} / \mathrm{Ac}_{2} \mathrm{O}$ to produce the corresponding $\beta$-bromoenol acetates in high regiolectivity are depicted in Table 2 entries 5-8. The reactions gave good to excellent yield with exception of phenylacetylene that formed, in addition to the desired $\beta$-haloenol acetate, the corresponding $\alpha, \alpha$-dibromocarbonyl product (formed from the hydrolyzed product) as well as the ring-brominated products (Scheme 4). Attempts to improve the formation of the $\beta$-bromoenol acetate by lowering the reaction temperature to $15^{\circ} \mathrm{C}$ resulted in no change in the reaction outcome. GC-MS monitoring of the reaction confirms that those by-products are formed from its inception, which demands further product column purification. After purification, the mixture of the stereoisomers, which we could not separate, was obtained in $51 \%$ yield (Table 2 , entry 6 ). On the other hand the $\beta$-bromoenol acetate derived from 3 -hexyne, was obtained as a single stereoisomer, indicating the corresponding bromonium ion as reaction intermediate. Attempts of purifying this compound by column chromatography resulted in degradation (Table 2 , entry 8 ). 
Similar results were obtained by reacting the alkynes with TCCA (Table 2, entries 1-4). Once more, using phenylacetylene as substrate, $\alpha, \alpha$-dichloroacetophenone and ring chlorination products were also observed (Scheme 4). The reaction with 1-phenyl-1-butyne was purified by flash chromatography, also affording the mixture of stereoisomers. The reaction of diphenylacetylene only resulted in the mixture of diasteroisomers, with no need of further purification (Table 2, entry 3). Attempts of purifying the product from 3-hexyne (Table 2 , entry 4) by flash chromatography failed, since it degrades on column.

Finally, the reaction of alkynes with TICA also led to the corresponding $\beta$-iodoenol acetates, but the reactions are much slower as shown by the reaction times in Table 2, entries 9-12. This led us to carry out most of the reaction in neat HOAc. Therefore, 1-phenyl-2-iodovinylacetate was efficiently obtained after $4 \mathrm{~h}$ of reaction as demonstrated by NMR analysis. However, upon isolation, a rapid color change of the product to violet was observed. Purification attempts of this product by column chromatography were unsuccessful, since degradation takes place. Reaction of TICA with phenylacetylene is cleaner, resulting on the corresponding $\beta$ iodoenol acetate after $3 \mathrm{~h}$ of reaction. The reaction product was found to be more stable, since it presented

Table 2. Monohalogenation of different alkynes with TXCA

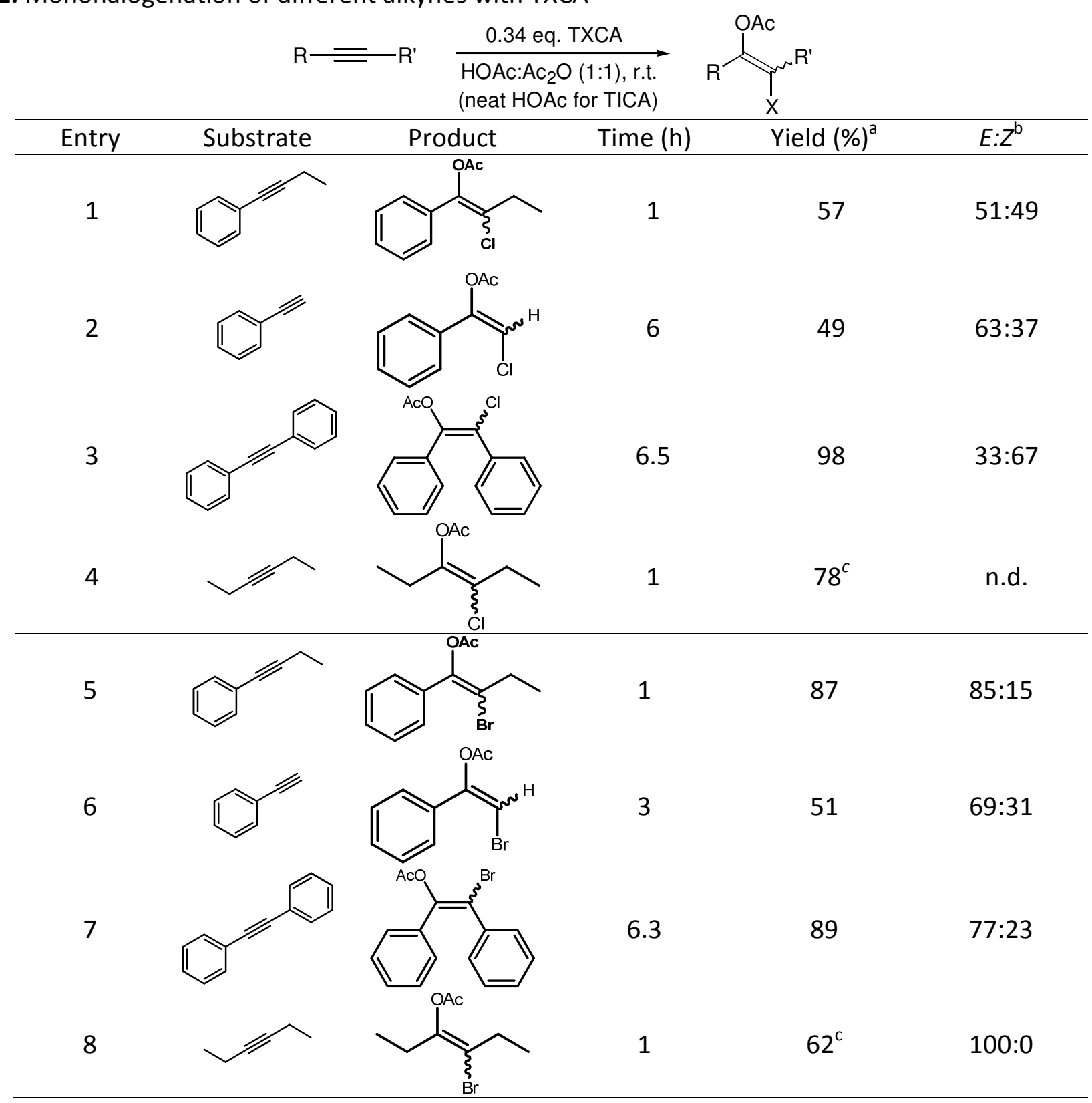


9<smiles>C#Cc1ccccc1</smiles>

10<smiles>CCC#Cc1ccccc1</smiles>

$11^{b}$

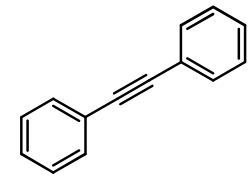<smiles>CC(=O)O/C(=C/I)c1ccccc1</smiles><smiles>CCC(C)=C(OC(C)=O)c1ccccc1</smiles><smiles>CO/C(=C(\I)c1ccccc1)c1ccccc1</smiles><smiles>CCC(C)=C(CC)C(=O)O</smiles>

4

3

16

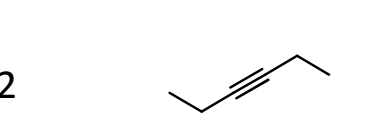

12

${ }^{a}$ Isolated yield; ${ }^{b}$ Determined by GC-MS; ${ }^{c}$ crude; ${ }^{d}$ reaction at $0{ }^{\circ} \mathrm{C}$.

satisfactory purity after reaction workup (Table 2, entry 9). The synthesis of the 1,2-diphenyl-2-iodoenol acetate from diphenylacetylene was initially carried out under the same conditions as the previous iodination reactions, but under these condition and 14 days, there still was starting material left as well as considerable quantities of benzil. To prevent formation of this product, the reaction was carried out using the 1:1 $\mathrm{HOAc}: \mathrm{Ac}_{2} \mathrm{O}$ mixture at $0{ }^{\circ} \mathrm{C}$, leading to a decrease of the amounts of benzil from $29 \%$ to $4 \%$. After flash chromatography, 34\% yield of the mixture of stereoisomers was obtained (Table 2, entry 10). Once again the reaction of TICA with 3-hexyne affording the 4-iodo-3-acetoxy-3-hexene was obtained in 79\% yield (crude) and $84 \%$ purity (table 2 , entry 12 ). Its purification by flash chromatography was not possible, since it degradates on column.

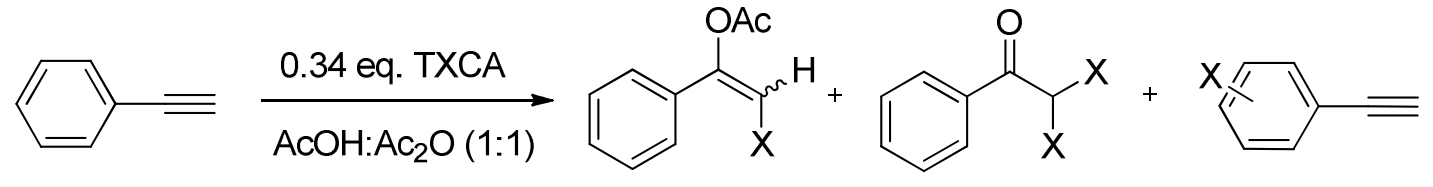

$$
\begin{array}{llll}
X=\mathrm{Cl} & 83 \% & 3 \% & 14 \% \\
\mathrm{X}=\mathrm{Br} & 68 \% & 18 \% & 14 \%
\end{array}
$$

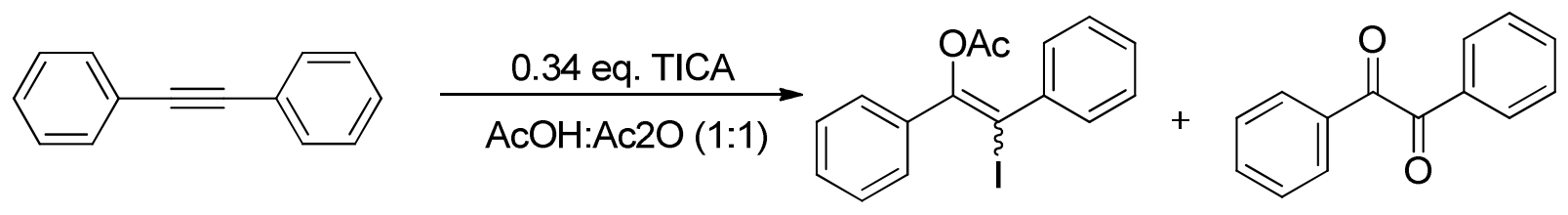

\section{Scheme 4}

\section{Conclusions}

We have shown that $\beta$-haloenol acetates can be efficiently obtained by reacting alkynes with trihaloisocyanuric acids in $\mathrm{HOAc}, \mathrm{Ac}_{2} \mathrm{O}$ or a mixture of these solvents. A mixture of $Z$ and $E$ stereoisomers of 
the corresponding $\beta$-haloenol acetate is generally obtained for conjugated alkynes, indicating the intermediacy of resonance stabilized vinyl cations, instead of the corresponding halonium ions in this case.

\section{Experimental Section}

General. TCCA and the alkynes were purchased from Sigma-Aldrich and used as received, while TBCA and TICA were synthesized according to the procedure previously reported by us. ${ }^{7,8}$ Solvents were purchased from Tedia and Vetec, and used without purification, unless otherwise stated.

NMR spectra were recorded on a Bruker spectrometer models AC-200 or AC-300. The spectra of NOESY-1D, NOESY-2D, COSY, HETCOR (in Supporting Information), as well as some ${ }^{1} \mathrm{H}$ and ${ }^{13} \mathrm{C}$ NMR analyses were obtained in a Varian 600-NMR. HRGC-MS analyses were performed on a Shimadzu GCMS-QP2010S gas chromatograph with electron impact (70 eV) by using a $30 \mathrm{~m}$ DB-5 silica capillary column. Diastereoisomeric ratios were determined by GC-MS, and the major component was determined by NMR.

Flash chromatography was carried out for purifying the products using a 15-20 mm diameter column, with 25 $\mathrm{cm}$ of silica gel (230-400 mesh) and 5\% ethyl acetate:hexane mixture as eluent.

General procedure. In a $10 \mathrm{~mL}$ round bottom flask, $0.34 \mathrm{mmol}$ of TXCA was added to a stirred mixture of $6 \mathrm{~mL}$ of solvent and the substrate (HOAc for iodinations and 1:1 (v/v) mixture of $\mathrm{HOAC}_{\mathrm{Ac}} \mathrm{O}$ for the chlorination and brominations). GC-MS analyses were carried out to evaluate the extension of the reaction and, after its completion; $10 \mathrm{~mL}$ of distilled water were added to the reaction medium, followed by addition of of $10 \%$ $\mathrm{NaHSO}_{3}(10 \mathrm{~mL})$. The aqueous phase was then extracted with ethyl acetate $(1 \times 20 \mathrm{~mL}+2 \times 10 \mathrm{~mL})$ and the combined organic phase was washed with a saturated aqueous solution of $\mathrm{NaHCO}_{3}$, dried $\left(\mathrm{Na}_{2} \mathrm{SO}_{4}\right)$, filtered and evaporated under vacuum (rotatory evaporator). The crude products were obtained by flash chromatography. NMR and MS analyses were carried out.

2-Bromo-1-phenylbut-1-en-1-yl acetate. ${ }^{16} \mathrm{C}_{12} \mathrm{H}_{13} \mathrm{BrO}_{2} ; \mathrm{MM} 269$; pale yellow oil. Yield: 87\% (468.1 mg; mixture of diastereoisomers $E: Z=88: 12)$. IR $(\mathrm{KBr}) \vee / \mathrm{cm}^{-1}: 3057,2976,2938,1765,1981,1200,1088,1057,1021,699$ ${ }^{1} \mathrm{H}$ NMR $\left(300 \mathrm{MHz}, \mathrm{CDCl}_{3}\right): \delta$ diastereoisomer $\left.E\right)=1.22-1.29\left(\mathrm{t}, \mathrm{J}=8.0 \mathrm{~Hz}, 3 \mathrm{H}, \mathrm{CH}_{3}\right) ; 2.14\left(\mathrm{~s}, 3 \mathrm{H}, \mathrm{COCH}_{3}\right) ; 2.58-$ $2.69\left(\mathrm{q}, J=8.0 \mathrm{~Hz}, 2 \mathrm{H}, \mathrm{CH}_{2}\right) ; 7.36-7.44(\mathrm{~m}, \mathrm{H} 3,3 \mathrm{Ar}-\mathrm{H}) ; 7.59-7.64(\mathrm{~m}, \mathrm{H} 2,2 \mathrm{Ar}-\mathrm{H})$. $\delta$ diastereoisomer $\left.\mathrm{Z}\right)=1.29-1.39$ $\left(\mathrm{t}, \mathrm{J}=8 \mathrm{~Hz}, 3 \mathrm{H}, \mathrm{CH}_{3}\right) ; 2.19\left(\mathrm{~s}, 3 \mathrm{H}, \mathrm{COCH}_{3}\right) ; 2.69-2.83\left(\mathrm{q}, \mathrm{J}=8.0 \mathrm{~Hz}, 2 \mathrm{H}, \mathrm{CH}_{2}\right) ; 7.36-7.44(\mathrm{~m}, \mathrm{H} 3,3 \mathrm{Ar}-\mathrm{H}) ; 7.59-7.64$ $(\mathrm{m}, \mathrm{H} 2,2 \mathrm{Ar}-\mathrm{H}) .{ }^{13} \mathrm{C} \mathrm{NMR}\left(75 \mathrm{MHz}, \mathrm{CDCl}_{3}\right): \delta$ diastereoisomer $\left.\mathrm{E}\right)=12.9\left(\mathrm{CH}_{3}\right) ; 20.8\left(\mathrm{CH}_{3}\right) ; 29.0\left(\mathrm{CH}_{2}\right) ; 120.9(\mathrm{C})$; 128.0 (m- $\mathrm{CH}) ; 128.9$ (p- $\mathrm{CH}) ; 129.4\left({ }_{0} \mathrm{CH}\right) ; 135.5\left(\mathrm{C}_{\mathrm{Ar}}\right) ; 143.3(\mathrm{C}) ; 168.5(\mathrm{CO}) . \delta$ diastereoisomer $\left.E\right)=13.8\left(\mathrm{CH}_{3}\right)$; $20.8\left(\mathrm{CH}_{3}\right) ; 29.5\left(\mathrm{CH}_{2}\right) ; 121.5$ (C); $128.6(\mathrm{~m}-\mathrm{CH}) ; 128.7$ (o- $\left.\mathrm{CH}\right) ; 129.2$ (p-CH); $134.2\left(\mathrm{C}_{\mathrm{Ar}}\right) ; 144.3$ (C); 168.1 (CO). MS $m / z$ (\%): 226 (59\%) and 228 (53\%), 211 (48\%) and 213 (46\%), 189 (100\%), 147 (25\%) 132 (33\%), 105 (28\%), 77 (67\%), 43 (68\%).

2-Bromo-1-phenylvinyl acetate. ${ }^{17} \mathrm{C}_{10} \mathrm{H}_{9} \mathrm{BrO}_{2} ; \mathrm{MM} 241$; pale yellow oil. Yield: $51 \%$ (245.8 mg; mixture of diastereoisomers $E: Z=69: 31)$. IR $(\mathrm{KBr}) \vee / \mathrm{cm}^{-1}: 3092,1760,1250,1164,1056,697 .{ }^{1} \mathrm{H} \mathrm{NMR}\left(600 \mathrm{MHz} \mathrm{CDCl}_{3}\right)$ : $\delta$ diastereoisomer $E)=2.19\left(\mathrm{~s}, 3 \mathrm{H}, \mathrm{CH}_{3}\right) ; 6.35(\mathrm{~s}, 1 \mathrm{H}, \mathrm{CH}) ; 7.37-7.43\left(\mathrm{~m}, \mathrm{H}_{\mathrm{Ar}}\right) ; 7.66-7.67\left(\mathrm{~m}, \mathrm{H}_{\mathrm{o}}\right) . \delta$ diastereoisomer Z) $=2.36\left(\mathrm{~s}, 3 \mathrm{H}, \mathrm{CH}_{3}\right) ; 6.58(\mathrm{~s}, 1 \mathrm{H}, \mathrm{CH}) ; 7.37-7.43\left(\mathrm{~m}, \mathrm{H}_{\mathrm{Ar}}\right) ; 7.66-7.67\left(\mathrm{~m}, \mathrm{H}_{\mathrm{o}}\right) .{ }^{13} \mathrm{C} \mathrm{NMR}(150$ $\left.\mathrm{MHz}, \mathrm{CDCl}_{3}\right)$ : $\delta$ diastereoisomer $\left.E\right)=20.9\left(\mathrm{CH}_{3}\right) ; 97.8(\mathrm{CH}) ; 128.3\left(\mathrm{CH}_{\mathrm{o}}\right) ; 128.4\left(\mathrm{CH}_{\mathrm{m}}\right) ; 129.6\left(\mathrm{CH}_{\mathrm{p}}\right) ; 133.4\left(\mathrm{C}_{\mathrm{Ar}}\right)$; $148.9(\mathrm{C}) ; 168.8$ (CO). $\delta$ diastereoisomer Z) = $20.7\left(\mathrm{CH}_{3}\right) ; 96.7(\mathrm{CH}) ; 125.0\left(\mathrm{CH}_{\mathrm{o}}\right) ; 128.9\left(\mathrm{CH}_{\mathrm{m}}\right) ; 134.1\left(\mathrm{C}_{\mathrm{Ar}}\right) ; 150.6$ (C); 167.4 (CO). MS m/z (\%): 198 (77\%) and 200 (72\%), 161 (56\%), 120 (17\%) and 122 (16\%), 78 (68\%), 77 (23\%), 51 (21\%), 43 (100). 
2-Bromo-1,2-diphenylvinyl acetate. ${ }^{18} \mathrm{C}_{16} \mathrm{H}_{13} \mathrm{BrO}_{2} ; \mathrm{MM} 317$; pale yellow solid. Yield: 89\% (564.3 mg; mixture of diastereoisomers $E: Z=72: 28)$. IR $(\mathrm{KBr}) \vee / \mathrm{cm}^{-1}:$ 3099, 3081, 3055, 3034, 1762, 1640, 1444, 1194, 1178, 1061, 1023, 756, 700. ${ }^{1} \mathrm{H} \operatorname{NMR}\left(200 \mathrm{MHz}, \mathrm{CDCl}_{3}\right): \delta$ diastereoisomer $\left.E\right)=2.00\left(\mathrm{~s}, 3 \mathrm{H}, \mathrm{CH}_{3}\right) ; 7.31-7.81\left(\mathrm{~m}, \mathrm{H}_{\mathrm{Ar}}\right) . \delta$ diastereoisomer $\mathrm{Z})=2.39\left(\mathrm{~s}, 3 \mathrm{H}, \mathrm{CH}_{3}\right) ; 7.31-7.81\left(\mathrm{~m}, \mathrm{H}_{\mathrm{Ar}}\right){ }^{13} \mathrm{C} \mathrm{NMR}\left(50 \mathrm{MHz}, \mathrm{CDCl}_{3}\right)$ : $\delta$ diastereoisomer $\left.E\right)=20.7$ $\left(\mathrm{CH}_{3}\right) ; 114.2(\mathrm{C}) ; 128.2\left(\mathrm{CH}_{\mathrm{m}}\right) ; 128.4\left(\mathrm{CH}_{\mathrm{m}}\right) ; 128.8\left(\mathrm{CH}_{\mathrm{p}}\right) ; 128.9\left(\mathrm{CH}_{\mathrm{o}}\right) ; 129.3\left(\mathrm{CH}_{\mathrm{o}}\right) ; 135.5\left(\mathrm{C}_{\mathrm{Ar}}\right) ; 146.1(\mathrm{C}) ; 168.7$ (CO). $\delta$ diastereoisomer Z) =21.0 ( $\left.\mathrm{CH}_{3}\right) ; 115.2(\mathrm{C}) ; 128.2\left(\mathrm{CH}_{\mathrm{m}}\right) ; 128.4\left(\mathrm{CH}_{\mathrm{m}}\right) ; 128.8\left(\mathrm{CH}_{\mathrm{p}}\right) ; 129.3\left(\mathrm{CH}_{\mathrm{o}}\right) ; 130.4$ $\left(\mathrm{CH}_{\mathrm{O}}\right) ; 134.1\left(\mathrm{C}_{\mathrm{Ar}}\right) ; 144.7(\mathrm{C}) ; 168.0(\mathrm{CO}) . \mathrm{MS} \mathrm{m} / \mathrm{z}(\%): 316\left(\mathrm{M}^{+} ; 0.1 \%\right)$ and $318\left((\mathrm{M}+2)^{+} ; 0.1 \%\right), 274(84 \%)$ and 276 (81\%), 237 (78\%), 194 (23\%), 165 (66\%), 105 (43\%), 77 (54\%), 51 (18\%), 43 (110\%).

4-Bromohex-3-en-3-yl acetate. $\mathrm{C}_{8} \mathrm{H}_{13} \mathrm{BrO}_{2} ; \mathrm{MM} 221$; pale yellow oil. Yield: $62 \%$ (274.0 mg; crude) ${ }^{1} \mathrm{H}$ NMR (200 $\mathrm{MHz}_{\mathrm{CDCl}}$ ): $\delta=0.98-10.60\left(\mathrm{t}, J=7.3 \mathrm{~Hz}, 3 \mathrm{H}, \mathrm{CH}_{3}\right) ; 1.02-1.09\left(\mathrm{t}, J=7.5 \mathrm{~Hz}, 3 \mathrm{H}, \mathrm{CH}_{3}\right) ; 2.17\left(\mathrm{~s}, 3 \mathrm{H}, \mathrm{CH}_{3}\right) ; 2.29-2.40$ $\left(q, J=7.3,2 \mathrm{H}, \mathrm{CH}_{2}\right) ; 2.43-2.54\left(\mathrm{q}, J=7.5 \mathrm{~Hz}, 2 \mathrm{H}, \mathrm{CH}_{2}\right) .{ }^{13} \mathrm{C} \mathrm{NMR}\left(50 \mathrm{MHz}, \mathrm{CDCl}_{3}\right): \delta=10.8\left(\mathrm{CH}_{3}\right) ; 12.8\left(\mathrm{CH}_{3}\right) ; 20.7$ $\left(\mathrm{CH}_{3}\right) ; 26.4\left(\mathrm{CH}_{2}\right) ; 28.1\left(\mathrm{CH}_{2}\right) ; 119.2$ (C); 146.9 (C); 168.7 (CO). MS - m/z (\%): $178(40 \%)$ and $180(38 \%), 163$ (36\%) and $165(33 \%), 141(24 \%), 99(21 \%), 55(26 \%), 43(100 \%)$.

2-Chloro-1-phenylvinyl acetate. ${ }^{19} \mathrm{C}_{10} \mathrm{H}_{9} \mathrm{ClO}_{2} ; \mathrm{MM} 196 \mathrm{~g} / \mathrm{mol}$, pale yellow oil. Yield: 49\% (192.0 mg; mixture of diastereoisomers $E: Z=63: 37)$. IR $(\mathrm{KBr}) \vee / \mathrm{cm}^{-1}: 3515,3086,2932,1770,1493,1446,1370,1202,1180,1064$, $1029,754,697 .{ }^{1} \mathrm{H}$ NMR $\left(600 \mathrm{MHz}, \mathrm{CDCl}_{3}\right): \delta$ diastereoisomer $\left.E\right)=2.21\left(\mathrm{~s}, 3 \mathrm{H}, \mathrm{CH}_{3}\right) ; 6.27(\mathrm{~s}, 1 \mathrm{H}, \mathrm{CH}) ; 7.37-7.43$ $\left(\mathrm{m}, \mathrm{H}_{\mathrm{Ar}}\right) ; 7.65-7.66\left(\mathrm{~m}, \mathrm{H}_{\mathrm{o}}\right) . \delta$ diastereoisomer $\left.\mathrm{Z}\right)=2.35\left(\mathrm{~s}, 3 \mathrm{H}, \mathrm{CH}_{3}\right) ; 6.47(\mathrm{~s}, 1 \mathrm{H}, \mathrm{CH}) ; 7.37-7.43\left(\mathrm{~m}, \mathrm{H}_{\mathrm{Ar}}\right) ; 7.65-$ $7.66\left(\mathrm{~m}, \mathrm{H}_{\mathrm{o}}\right) .{ }^{13} \mathrm{C} \mathrm{NMR}\left(150 \mathrm{MHz}, \mathrm{CDCl}_{3}\right): \delta$ diastereoisomer $\left.\mathrm{E}\right)=21.0\left(\mathrm{CH}_{3}\right) ; 110.6(\mathrm{CH}) ; 128.1\left(\mathrm{CH}_{\mathrm{o}}\right) ; 128.5$ $\left(\mathrm{CH}_{\mathrm{m}}\right) ; 129.6\left(\mathrm{CH}_{\mathrm{p}}\right) ; 132.4\left(\mathrm{C}_{\mathrm{Ar}}\right) ; 148.0(\mathrm{C}) ; 169.1(\mathrm{CO}) . \delta$ diastereoisomer Z) = $20.7\left(\mathrm{CH}_{3}\right) ; 108.2(\mathrm{CH}) ; 124.9$ $\left(\mathrm{CH}_{\mathrm{o}}\right) ; 129.0\left(\mathrm{CH}_{\mathrm{m}}\right) ; 129.6\left(\mathrm{CH}_{\mathrm{p}}\right) ; 133.0\left(\mathrm{C}_{\mathrm{Ar}}\right) ; 148.7(\mathrm{C}) ; 167.6(\mathrm{CO}) . \mathrm{MS}-\mathrm{m} / \mathrm{z}(\%): 196\left(\mathrm{M}^{+} ; 2.5 \%\right)$ and 198 $\left((\mathrm{M}+2)^{+} ; 0.8 \%\right), 154(52 \%)$ and $156(17 \%), 105(5 \%), 78(66 \%), 77(20 \%), 51(17 \%), 43(100 \%)$.

2-Chloro-1-phenylbut-1-en-1-yl acetate. ${ }^{16,20} \mathrm{C}_{12} \mathrm{H}_{13} \mathrm{ClO}_{2} ; \mathrm{MM}$ 224, pale yellow oil. Yield: $57 \%$ (255.4 mg; mixture of diastereoisomers $E: Z=51: 49){ }^{1} \mathrm{H} \mathrm{NMR}\left(200 \mathrm{MHz}, \mathrm{CDCl}_{3}\right): \delta$ diastereoisomer $\left.E\right)=1.18(\mathrm{t}, J=8.0 \mathrm{~Hz}$, $\left.3 \mathrm{H}, \mathrm{CH}_{3}\right) ; 2.15\left(\mathrm{~s}, 3 \mathrm{H}, \mathrm{CH}_{3}\right) ; 2.17-2.51\left(\mathrm{q}, \mathrm{J}=8.0 \mathrm{~Hz}, 2 \mathrm{H}, \mathrm{CH}_{2}\right) ; 7.31-7.39(\mathrm{~m}, 5 \mathrm{Ar}-\mathrm{H})$. $\delta$ diastereoisomer $\left.\mathrm{Z}\right)=1.25$ $\left(\mathrm{t}, \mathrm{J}=8 \mathrm{~Hz}, 3 \mathrm{H}, \mathrm{CH}_{3}\right) ; 2.17\left(\mathrm{~s}, 3 \mathrm{H}, \mathrm{CH}_{3}\right) ; 2.15-2.49\left(\mathrm{q}, \mathrm{J}=8.0 \mathrm{~Hz}, 2 \mathrm{H}, \mathrm{CH}_{2}\right) ; 7.31-7.39(\mathrm{~m}, 5 \mathrm{Ar}-\mathrm{H}) .{ }^{13} \mathrm{C} \mathrm{NMR}(50 \mathrm{MHz}$, $\left.\mathrm{CDCl}_{3}\right): \delta$ diastereoisomers $E$ and $\left.\mathrm{Z}\right)=12.8$ and $11.9\left(\mathrm{CH}_{3}\right) ; 20.7$ and $20.8\left(\mathrm{CH}_{3}\right) ; 29.9$ and $27.8\left(\mathrm{CH}_{2}\right), 128.1$ and $128.6\left(\mathrm{CH}_{\mathrm{m}}\right) ; 128.8$ and $128.9\left(\mathrm{CH}_{\mathrm{o}}\right) ; 129.1\left(\mathrm{CH}_{\mathrm{p}}\right) ; 134.3$ and $134.1(\mathrm{C}) ; 168.2$ and $168.7(\mathrm{CO}) . \mathrm{MS} \mathrm{m} / \mathrm{z}(\%): 224$ $\left(\mathrm{M}^{+} ; 1.5 \%\right)$ and $\left.226(\mathrm{M}+2)^{+} ; 0.5 \%\right), 189(19 \%), 182(65 \%)$ and $184(21 \%), 167(54 \%)$ and $169(18 \%), 147(16 \%)$, 131 (23\%), 105 (23\%), 89 (23\%), 77 (63\%), 51 (25\%), 43 (100\%).

2-Chloro-1,2-diphenylvinyl acetate. ${ }^{18,20} \mathrm{C}_{16} \mathrm{H}_{13} \mathrm{ClO}_{2} ; \mathrm{MM} \mathrm{272,} \mathrm{pale} \mathrm{yellow} \mathrm{solid.} \mathrm{Yield:} \mathrm{98 \%} \mathrm{(533.1} \mathrm{mg;} \mathrm{mixture}$ of diastereoisomers $E: Z=33: 67){ }^{1} \mathrm{H}$ NMR $\left(200 \mathrm{MHz}, \mathrm{CDCl}_{3}\right): \delta$ diastereoisomer $\left.Z\right)=2.31\left(\mathrm{~s}, 3 \mathrm{H}, \mathrm{CH}_{3}\right) ; 7.21-7.73$ $\left(\mathrm{m}, 10 \mathrm{H}, \mathrm{H}_{\mathrm{Ar}}\right) . \delta$ diastereoisomer $\left.E\right)=1.98\left(\mathrm{~s}, 3 \mathrm{H}, \mathrm{CH}_{3}\right) ; 7.21-7.73\left(\mathrm{~m}, 10 \mathrm{H}, \mathrm{H}_{\mathrm{Ar}}\right) .{ }^{13} \mathrm{C} \mathrm{NMR}\left(50 \mathrm{MHz}^{\mathrm{CDCl}}\right)_{3}: \delta$ (diastereoisomer Z) = $20.9\left(1 \mathrm{CH}_{3}\right) ; 124.2(1 \mathrm{C}) ; 128.3\left(2 \mathrm{CH}_{\mathrm{m}}\right) ; 128.5\left(2 \mathrm{CH}_{\mathrm{m}}\right) ; 129.0\left(2 \mathrm{CH}_{\mathrm{o}}\right) ; 129.3\left(2 \mathrm{CH}_{\mathrm{o}}\right) ; 130.1$ $\left(2 \mathrm{CH}_{\mathrm{p}}\right) ; 134.4\left(1 \mathrm{C}_{\mathrm{Ar}}\right) ; 136.2\left(1 \mathrm{C}_{\mathrm{Ar}}\right) ; 144.4(1 \mathrm{C}) ; 168.1(1 \mathrm{CO}) . \delta$ (diastereoisomer $\left.E\right)=20.9\left(1 \mathrm{CH}_{3}\right) ; 124.0$ $(1 \mathrm{C}) ; 128.3\left(2 \mathrm{CH}_{\mathrm{m}}\right) ; 128.5\left(2 \mathrm{CH}_{\mathrm{m}}\right) ; 129.0\left(4 \mathrm{CH}_{\mathrm{o}}\right) ; 130.1\left(2 \mathrm{CH}_{\mathrm{p}}\right) ; 128.8\left(2 \mathrm{CH}_{\mathrm{p}}\right) ; 134.1\left(1 \mathrm{C}_{\mathrm{Ar}}\right) ; 136.5\left(1 \mathrm{C}_{\mathrm{Ar}}\right) ; 143.6$ (1C); 168.9 (1CO). MS m/z (\%): $272\left(\mathrm{M}^{+} ; 3.3 \%\right)$ and $\left.274(\mathrm{M}+2)^{+} ; 1.2 \%\right), 230(100 \%)$ and $232(32 \%), 195(10 \%)$, 165 (40\%), 152 (30\%), 124 (32\%), 105 (28\%), 89 (20\%), 77 (50\%), 51 (15\%), 43 (74\%).

4-Chlorohex-3-en-3-yl acetate: $\mathrm{C}_{8} \mathrm{H}_{13} \mathrm{ClO}_{2} ; \mathrm{MM} \mathrm{176,} \mathrm{pale} \mathrm{yellow} \mathrm{oil.} \mathrm{Yield:} \mathrm{78 \%} \mathrm{(274.6mg;} \mathrm{crude).} \mathrm{MS} \mathrm{-} \mathrm{m} / z$ (\%): 176 and $178(\mathrm{M}+$ and $(\mathrm{M}+2)+/ 0.4$ and $0.14 \%), 141(20 \%), 134$ and 136 (53 and 17.5\% ), 119 and 121 (64 and 21.5\%), 99 (23\%), 55 (26\%), $43(100 \%)$.

2-lodo-1-phenylvinyl acetate. ${ }^{19,21} \mathrm{C}_{10} \mathrm{H}_{9} \mathrm{IO}_{2} ; \mathrm{MM}: 288$, pale yellow oil, degrade. Yield: $40 \%$. MS - $m / z(\%): 288$ (1\%), 246 (100\%), 168 (29\%), 161 (27\%), 105 (17\%), 91 (19\%), 77 (15\%), 51 (16\%), 43 (66\%).

(E)-2-lodo-1-phenylbut-1-en-1-yl acetate. $\mathrm{C}_{12} \mathrm{H}_{13} \mathrm{IO}_{2} ; \mathrm{MM} \mathrm{316,} \mathrm{pale} \mathrm{yellow} \mathrm{oil.} \mathrm{Yield:} \mathrm{86 \%} \mathrm{(543.5} \mathrm{mg).} \mathrm{IR} \mathrm{(KBr)} \mathrm{v}$ $/ \mathrm{cm}^{-1}: 3056,2972,1762,1350,1198,1082,1050,1018,699 .{ }^{1} \mathrm{H}$ NMR $\left(600 \mathrm{MHz} \mathrm{CDCl}_{3}\right): \delta=1.14-1.17(\mathrm{t}, \mathrm{J}=\mathrm{Hz}$, 
$\left.3 \mathrm{H}, \mathrm{CH}_{3}\right) ; 2.13\left(\mathrm{~s}, 3 \mathrm{H}, \mathrm{CH}_{3}\right) ; 2.57-2.61\left(\mathrm{q}, \mathrm{J}=\mathrm{Hz}, 2 \mathrm{H}, \mathrm{CH}_{2}\right) ; 7.34-7.51\left(\mathrm{~m}, 5 \mathrm{H}, \mathrm{H}_{\mathrm{Ar}}\right) .{ }^{13} \mathrm{C} \mathrm{NMR}(150 \mathrm{MHz}, \mathrm{CDCl} 3): \delta=$ $14.2\left(\mathrm{CH}_{3}\right) ; 20.7\left(\mathrm{CH}_{3}\right) ; 32.2\left(\mathrm{CH}_{2}\right) ; 98.5(\mathrm{C}) ; 128.1\left(\mathrm{CH}_{\mathrm{m}}\right) ; 129.1\left(\mathrm{CH}_{\mathrm{p}}\right) ; 129.9\left(\mathrm{CH}_{\mathrm{o}}\right) ; 137.6\left(\mathrm{C}_{\mathrm{Ar}}\right) ; 145.9(\mathrm{C}) ; 168.5$ (CO). MS m/z (\%): 274(97\%), 259 (58\%), 189 (100\%), 147 (23\%), 132 (60\%), 115 (26\%), 105 (45\%), 77 (94\%), 69 (72\%), 51 (36\%), 43 (100\%).

2-lodo-1,2-diphenylvinyl acetate. ${ }^{19,21} \mathrm{C}_{16} \mathrm{H}_{13} \mathrm{IO}_{2} ; \mathrm{MM} 364$, pale yellow oil. Yield: $34 \%$ (247.5 mg; mixture of diastereoisomers $E: Z=87: 13)$. ${ }^{1} \mathrm{H} \mathrm{NMR}\left(200 \mathrm{MHz}, \mathrm{CDCl}_{3}\right): \delta$ diastereoisomer $\left.E\right)=1.75\left(\mathrm{~s}, 3 \mathrm{H}, \mathrm{CH}_{3}\right) ; 7.06-7.92$ $\left(\mathrm{m}, 10 \mathrm{H}, \mathrm{H}_{\mathrm{Ar}}\right) . \delta$ diastereoisomer $\left.\mathrm{Z}\right)=2.21\left(\mathrm{~s}, 3 \mathrm{H}, \mathrm{CH}_{3}\right) ; 7.06-7.92\left(\mathrm{~m}, 10 \mathrm{H}, \mathrm{H}_{\mathrm{Ar}}\right) . \mathrm{MS}-\mathrm{m} / \mathrm{z}(\%): 322(90 \%), 237$ (100\%), 195 (87\%), 177(33\%), 165 (75\%), 152 (21\%), 105 (14\%), $77(25 \%), 51(10 \%), 43$ (62\%).

4-lodohex-3-en-3-yl acetate. ${ }^{19,22} \mathrm{C}_{8} \mathrm{H}_{13} \mathrm{IO}_{2} ; \mathrm{MM} 268$, pale yellow oil. Yield: 79\% (423.4 mg). ${ }^{1} \mathrm{H} \mathrm{NMR}(200 \mathrm{MHz}$, $\left.\mathrm{CDCl}_{3}\right): \delta=0.98-1.04\left(\mathrm{t}, 6 \mathrm{H}, 2 \mathrm{CH}_{3}\right) ; 2.17\left(\mathrm{~s}, 3 \mathrm{H}, \mathrm{CH}_{3}\right) ; 2.30-2.36\left(\mathrm{q}, 2 \mathrm{H}, \mathrm{CH}_{2}\right) ; 2.51-2.58\left(\mathrm{q}, 2 \mathrm{H}, \mathrm{CH}_{2}\right) .{ }^{13} \mathrm{C} \mathrm{NMR}(50$ $\left.\mathrm{MHz}, \mathrm{CDCl}_{3}\right): \delta=11.0\left(\mathrm{CH}_{3}\right) ; 14.2\left(\mathrm{CH}_{3}\right) ; 20.6\left(\mathrm{CH}_{3}\right) ; 30.0\left(\mathrm{CH}_{2}\right) ; 31.4\left(\mathrm{CH}_{2}\right) ; 96.3(\mathrm{C}) ; 148.9$ (C); 168.5 (CO). MS $m / z(\%): 268\left(\mathrm{M}^{+}, 3 \%\right), 226(100 \%), 211(50 \%), 141(34 \%), 99(20 \%), 55(20 \%), 43$ (95\%).

\section{Acknowledgements}

We thank CNPq, CAPES and FAPERJ for financial support.

\section{Supplementary Material}

Spectral characterization of the products is available in the Supplementary File.

\section{References}

1. Mendonça, G. F.; de Almeida, L. S.; de Mattos, M. C. S.; Esteves, P. M.; Ribeiro, R. S. Curr. Org. Synth. 2015, 12, 603. https://doi.org/10.2174/157017941205150821130712

2. de Almeida, L. S.; Esteves, P. M.; de Mattos, M. C. S. Tetrahedron Lett. 2015, 56, 6843. https://doi.org/10.1016/i.tetlet.2015.10.081

3. Crespo, L. T. C.; Ribeiro, R. da S.; de Mattos, M. C. S.; Esteves, P. M. Synthesis 2010, 2379.

4. Fonseca, G.; Rejane, M.; Magalhaes, R.; de Mattos, M. C. S.; Esteves, P. M. J. Braz. Chem. Soc. 2005, 16, 695.

https://doi.org/10.1590/S0103-50532005000500003

5. de Almeida, L. S.; Esteves, P. M.; de Mattos, M. C. S. Curr. Green Chem. 2014, 1, 94. https://doi.org/10.2174/2213346101999140109142834

6. Mendonça, G. F.; de Mattos, M. C. S. Curr. Org. Synth. 2013, 10, 820. https://doi.org/10.2174/157017941006140206102255

7. de Almeida, L. S.; Esteves, P. M.; de Mattos, M. C. S. Synlett 2006, 1515.

8. Ribeiro, R.S.; Esteves, P.M.; de Mattos, M.C.S. Tetrahedron Lett. 2007, 48, 8747 https://doi.org/10.1016/i.tetlet.2007.10.011

9. de Almeida, L.S.; Esteves, P.M.; de Mattos, M.C.S. Synlett 2007, 1687.

10. Ribeiro, R.S.; Esteves, P.M.; de Mattos, M.C.S. J. Braz. Chem. Soc. 2012, 23, 228. 
11. Tozetti, S.D.F.; de Almeida, L.S.; Esteves, P.M.; de Mattos, M.C.S. J. Braz. Chem. Soc. 2007, $18,675$. https://doi.org/10.1590/S0103-50532007000400002

12. Gonzáles-Liste, P.J.; Francos, J.; García-Garrido, S.E.; Cadierno, V. J. Org. Chem. 2017, 82, 1507 and references cited therein.

https://doi.org/10.1021/acs.joc.6b02712

13. Barluenga, J.; Rodríguez, M. A.; Campos, P. J. J. Org. Chem. 1990, 55, 3104.

https://doi.org/10.1021/jo00297a027

14. Chen, Z.; Li, J.; Jiang, H.; Zhu, S.; Li, Y.; Qi, C. Org. Lett. 2010, 12, 3262. https://doi.org/10.1021/ol101251n

15. Chen, X.; Chen, D.; Lu, Z.; Kong, L.; Zhu, G. J. Org. Chem. 2011, 76, 6338. https://doi.org/10.1021/jo2005318

16. Shchepin, P. G.; Gladkova, V . V.; Neifel'd, G. E. Zhurnal Org. Khimii 1990, 26, 2394-2397.

17. Kowalski, C. J.; Haque, M. S. J. Org. Chem. 1985, 50, 5140-5142. https://doi.org/10.1021/jo00225a032

18. Chan, C.-K.; Chang, M.-Y. Synthesis 2016, 48, 3785-3793. https://doi.org/10.1055/s-0035-1561472

19. Barluenga, J. ; Martínez-Gallo, J. M.; Nájera, C.; Yus, M. J. Chem. Soc., Perkin Trans. 1 1987, $1017-1019$. https://doi.org/10.1039/P19870001017

20. Yates, K.; Go, T. A. J. Org. Chem. 1980, 45, 2377-2384. https://doi.org/10.1021/jo01300a023

21. Xia, X.-F.; Gu, Z.; Liu, W.; Wang, N.; Wang, H.; Xia, Y.; Gao, H.; Liu, X. Org. Biomol. Chem. 2014, 12, 99099913.

https://doi.org/10.1039/C4OB01404A

22. Barluenga, J.; Rodriguez, M. A.; Campos, P. J. J. Org. Chem. 1990, 55, 3104-3106. https://doi.org/10.1021/jo00297a027 